\title{
Getting to the bottom of right heart failure
}

\author{
Martin Koestenberger ${ }^{1,2}$, Harm-Jan Bogaard ${ }^{3}$, Georg Hansmann ${ }^{2,4}$ \\ ${ }^{1}$ Division of Pediatric Cardiology, Department of Pediatrics, Medical University Graz, Graz, Austria; ${ }^{2}$ European Pediatric Pulmonary Vascular \\ Disease Network, Berlin, Germany; ${ }^{3}$ Pulmonary Medicine, Amsterdam Cardiovascular Sciences, Amsterdam UMC, location VUmc, Amsterdam, \\ The Netherlands; ${ }^{4}$ Department of Pediatric Cardiology and Critical Care, Hannover Medical School, Hannover, Germany \\ Correspondence to: Martin Koestenberger, MD. Department of Pediatrics, Medical University Graz, Auenbruggerplatz 34/2, A-8036 Graz, Austria. \\ Email: martin.koestenberger@medunigraz.at.
}

Submitted Jun 13, 2020. Accepted for publication Aug 27, 2020.

doi: $10.21037 / \mathrm{cdt}-20-565$

View this article at: http://dx.doi.org/10.21037/cdt-20-565

In the last decades, substantial progress has been made in the diagnosis and treatment of right ventricular (RV) dysfunction in pediatric and adult patients (1-6). The pathobiology of right ventricular dysfunction (RVD), however, is heterogenous and broad, and, thus, there are many obstacles and areas of uncertainties to unpack (7-9). In this special issue, internationally renowned experts from multiple specialties comprehensively discuss impactful discoveries and innovations in the fascinating field of RV dysfunction that have emerged in the last years, both in adult and the pediatric disciplines.

The articles of this issue include reviews, commentaries, case reports and an original article, and all of them underwent rigorous external peer review. As special issue editors, we did not strive to fully cover all aspects of RV dysfunction but rather intended to create a compilation of papers that will be useful for the majority of pediatric and adult cardiologists, cardiac surgeons, pulmonologists, neonatologists, critical care physicians, physician scientists, and basic researchers with a particular interest in cardiovascular development, pulmonary vascular disease (PVD) and congenital heart disease (CHD), and ultimate heart failure and its molecular mechanisms/genetics. Each article was written by $2-11$ international experts from two or more institutions and countries, in order to encourage controversial discussions, reduce bias to once own work, and as such, achieve substantial scientific balance. We do express our sincere appreciation to the authors for their voluntary time and effort, and for the high quality that has been achieved in each manuscript.

In this issue, Agrawal et al. (10) provide novel insights into the role of "Molecular Mechanisms of RV Dysfunction and Heart Failure in Pulmonary Arterial Hypertension (PAH)", with a special focus on metabolism, angiogenesis, and sex hormones. The authors discuss the current understanding of key molecular mechanisms that cause, contribute and/or sustain RV dysfunction all the way to RV dilation and failure, with a special focus on pathways that can be targeted therapeutically. In particular, this review discusses the mechanisms by which vessel loss and dysfunctional angiogenesis, metabolic derangements, and sex hormones directly contribute to RV dysfunction in PAH. The authors highlight future areas of investigation that may lead to novel understanding and therapeutic interventions for RV dysfunction in $\mathrm{PAH}$.

In their comprehensive review article entitled "Left Ventricular Dysfunction in Heart Failure with Preserved Ejection Fraction (HFpEF) - Molecular Mechanisms and Impact on Right Ventricular Function", Heinzel et al. (11) discuss recent progress in understanding the epidemic condition HFpEF. The clinical and cellular phenotypes of HFpEF are explained and common co-morbidities, clinical phenotypes, as well as animal models of HFpEF are introduced. Moreover, the authors discuss the increased relevance of myocardial passive stiffness and active cardiomyocyte relaxation (e.g., altered cytosolic $\mathrm{Ca} 2+/ \mathrm{Na}+$ signaling) in this specific disease. Consistent with the overall topic of this special issue, they highlight RV dysfunction in HFpEF and provide possible explanations for $\mathrm{RV}$ dysfunction

Following the above mechanistic basic-translational science articles on $\mathrm{RV}$ and $\mathrm{LV}$ dysfunction and interdependence, Andersen et al. (12) give a very insightful State-of-the-Art review on "Animal models of Right Ventricular failure". After an introduction on animal 
models of RV failure, the authors discuss different modes of therapeutic targets, advantages and disadvantages in different species and models, and define RV failure in animal models. The writing group familiarizes us with the available pulmonary artery constriction/ligation models and present an overview of available models of pulmonary artery banding (alone or in combination with vascular endothelial growth factor receptor inhibition); they also present a new swine model of chronic thromboembolic $\mathrm{PH}$ (CTEPH). Finally, future directions of animal research on RV dysfunction, including the shortcomings of the available models, complement their review article (12).

In their superb state-of-the-art review article on "Mechanics of right ventricular dysfunction in pulmonary arterial hypertension and heart failure with preserved ejection fraction", Bernardo et al. (13) summarize and discuss recent knowledge of RV-pulmonary arterial (PA) coupling and adaptation of the hard-pressed RV. They take a closer look at the different mechanical components of RV dysfunction and ventricular dyssynchrony, followed by the insights into pressure-volume loop analysis, energetics and novel flow patterns such as vortex imaging. The authors also provide an in-depth comparison of prevalence and characteristics of RV dysfunction in HFpEF and $\mathrm{PH}$ (13).

Tremendous success has been made in pediatric and adult cardiology so that the majority of children with common or even complex CHD, with or without PAH, survive corrective or palliative surgery. Because of advances in the treatment of CHD, pediatric and adult cardiologists are now faced with a high number of adult survivors, socalled "grown-up's with CHD". Moreover, patients with $\mathrm{PAH}-\mathrm{CHD}$, alike those with idiopathic or heritable PAH, usually cannot be cured and their outcomes are determined by the ability of the RV to cope with alterations in cardiac structure, preload and afterload.

Truong et al. (14) provide a very comprehensive "Update on noninvasive imaging of right ventricular dysfunction in pulmonary hypertension". This review compiles the latest advancement in the noninvasive evaluation of pediatric and adult patients with $\mathrm{PH}$, by means of echocardiography, chest computed tomography, and cardiovascular magnetic resonance imaging. The authors explore how non-invasive parameters can support or sometimes even surpass the hemodynamic information that is provided with the calculated pulmonary vascular resistance (PVR) by cardiac catherization. The authors challenge the readers to question the "gold standard" therapeutic aim of reducing PVR as primary therapeutic target (14).
Santens et al. (15) review "Right ventricular dysfunction in Congenital Heart Disease (CHD)". They comprehensively describe how RV dysfunction in CHD can be detected and treated early in order to prevent or postpone fatal RV failure. Focusing on RV dysfunction in younger adults, the authors provide a description of anatomic and physiologic differences between the LV and the RV, and review the role of the biomarkers NT-proBNP (N-terminal pro-B-type natriuretic peptide), high-sensitive troponins, galectin-3 (Gal-3), and growth differentiation factor-15 (GDF-15) in assessing and monitoring RV dysfunction. Moreover, they describe changes in peak oxygen consumption that suggest RV deterioration. Finally, a vision for the future is given on the role of electrical optimization, mechanical circulatory support (MCS), and heart transplantation for advanced stages of RV dysfunction in CHD.

Sanders et al. (16) in their review "RV Dysfunction and Long-term Risk of sudden cardiac death" explore the unique role of RV dysfunction modifying long-term risk of adverse outcomes, and delineate the potential mechanisms that may underlie sudden cardiac death (SCD). They describe the resting anatomy of the RV and $\mathrm{LV}$ as well as anatomic changes in the RV and $\mathrm{LV}$ during the cardiac cycle, followed by describing the cardinal physiologic relationships governing normal and pathologic RV function (16). Changes in RV cavity dimension, contractility, and diastolic function have been described to occur commonly as a consequence of LV dysfunction, but are also welldocumented in isolated right coronary artery myocardial infarction, and less so in patients with primary $\mathrm{RV}$ cardiomyopathies including arrhythmogenic RV dysplasia. Finally, the authors summarize cardiac ischemia in the RV $v s$. in the $\mathrm{LV}$, chronic heart failure and post-capillary $\mathrm{PH}$ in the adult populations.

Inampudi et al. (17) discuss "Treatment of Right Ventricular Dysfunction and Heart Failure in pulmonary arterial bypertension patients". The multi-disciplinary authors point out the differences between a pressure overloaded $\mathrm{RV}$ and postcapillary $\mathrm{PH}$ due to $\mathrm{LV}$ diastolic dysfunction (e.g., HFpEF). They write on the current understanding of ventricular-ventricular interactions and highlight the interesting phenomenon of coronary malperfusion and ischemia in advanced stages of PAH and associated RV hypertrophy. After identification and management of triggering factors for RV dysfunction, and principles of intensive care unit monitoring they familiarize the reader with modern medical management, mechanical support of 
the heart up to the final stages of treatment (e.g., bilateral lung transplantation).

Schweintzger et al. (18) conducted a clinical, prospective pediatric study, and now publish new data on the "Safety and efficacy of off-label therapy with the endothelin receptor antagonist macitentan in pediatric pulmonary hypertension". The group is part of the European Pediatric Pulmonary Vascular Disease Network (EPPVDN) and investigatedfor the first time-the treatment effects of the dual endothelin receptor antagonist macitentan in infants and young children with $\mathrm{PH}$ under 12 years of age, in a prospective, observational fashion. After a median followup of 6 months, macitentan treatment, mostly add-on to monotherapy sildenafil, was associated with significant improvement of invasive hemodynamics (e.g., the ratio of mean pulmonary arterial pressure/mean systemic arterial pressure decreased from a median of $62 \%$ to $49 \%, \mathrm{P}<0.05$; pulmonary vascular resistance index (PVRi) decreased from a median of 7.6 to 4.8 Wood units $\times \mathrm{m}^{2}$ body surface area, $\mathrm{P}<0.05$ ), echocardiographic variables of RV function (e.g., tricuspid annular plane systolic excursion increased from a median of 1.4 to $1.9 \mathrm{~cm}, \mathrm{P}<0.05$ ), and the heart failure biomarker NT-pro-BNP. Importantly, macitentan therapy was well tolerated.

We are grateful to the clinical fellows and faculty who contributed very interesting case reports on PVD and RV dysfunction to this special issue.

Drs. Giagnorio and Hansmann (19) report on a preterm infant with bronchopulmonary dysplasia (BPD), severe $\mathrm{PH}$ and RV failure who greatly and rapidly improved by means of clinical status, blood biomarkers and echocardiography, following the addition of spironolactone and hydrochlorothiazide to the $\mathrm{PH}$-targeted treatment regimen (19).

Bakas et al. (20) present a case of an infant with RV dysfunction and $\mathrm{PH}$ due to prenatal ductal closure, who was successfully treated with milrinone with full recovery of cardiac function. Therefore, milrinone may be considered as a therapeutic agent particularly in such infants with RV dysfunction.

Sallmon et al. (21) report on the interventional creation of an "endogenous" reverse Potts shunt by stenting a preexisting small but patent ductus arteriosus (PDA) in a 2 months old female infant with severe, supra-systemic $\mathrm{PH}$, associated with a novel combination of a compound heterozygous $\mathrm{ABCA} 3$ mutation and heterozygous genetic variants of surfactant protein $\mathrm{B}$ and $\mathrm{C}$. This combination of human genetic mutations has not been described before in viable infants, children or adults, and adds to the novelty of the findings in this report.

Dejea et al. (22) wrote a fascinating review on the "Cardiac Multi-scale Investigation of the Right and Left Ventricle exvivo". The researchers suggest that only multiscale imaging approaches achieve a detailed description of the ventricular architecture and cardiac contractile function, necessary to fully understand the relationship between structural remodeling and cardiac dysfunction in cardiovascular diseases. The authors introduce techniques that are used to study ventricular architecture, i.e., ultrasound, optical computed tomography, (histological) optical techniques, magnetic resonance imaging, micro-computed tomography and, synchrotron $\mathrm{X}$-ray phase-contrast imaging, once of the most advanced, high resolution imaging technology. This review not only gives an overview about available or emerging cardiovascular imaging techniques for assessing myocardial architecture, but also discusses their main advantages and limitations and how they can be used to quantify cardiac remodeling at different scales in cardiovascular disease.

In their Editorial Commentary, Drs. Hansmann, Rich, and Maron (23), entitled "Cardiac Catheterization in Pulmonary Hypertension: Doing it Right, with a Catheter on the Left", propose that left heart catheterization, including direct measurement of LV-end-diastolic pressure (LVEDP), at best simultaneously with pulmonary arterial wedge pressure (PAWP), offers a diagnostic advantage to PAWP alone for identifying $\mathrm{PH}$ patients with either pulmonary venous hypertension, significant lung disease, or mitral stenosis. Coronary angiography may be indicated in selected cases when PH is confirmed. The authors suggest that overall, future consensus statements are needed that consider strengthening the indication for left heart catheterization in the diagnostic evaluation of $\mathrm{PH}$ patients by clarifying specific scenarios for which this approach is warranted (23).

Drs. Navaratnam and DiNardo (24) present their anesthesiologist's view on peri-operative RV dysfunction. They underline the importance of RV dysfunction and failure on peri-operative morbidity/mortality ad highlight the pathophysiology of RV failure and ventricular interdependencies. The authors set a special focus on perioperative management with impact of anesthetic medications, positive pressure ventilation, and RV support strategies, including inotropic/lusotropic agents and pulmonary vasodilators. Finally, the authors discuss pitfalls in weaning patients from the cardiopulmonary 
bypass and in the post-operative management (intubation versus extubation or positive pressure versus spontaneous ventilation).

Finally, Klinke et al. (25) in their interdisciplinary, very comprehensive review article on "Emerging Therapies for RV Dysfunction and Failure", summarize and discuss the latest developments in the field. These include current pharmacological, RNA- and cell-based therapeutics and their potential to directly target the RV. They also review the available study data for pulmonary artery denervation and mechanical circulatory support. The authors finally highlight important future research directions on RV function, dysfunction and failure.

Taken together, through innovate basic and clinical research on RV dysfunction, great progress has been made both in the refinement of diagnostic tools and treatment. With the contributions of a wonderful group of clinicians and scientists coauthoring the papers of this Cardiovascular Diagnosis and Therapy special issue, we hope to have gathered a collection of articles that will be of great interest to the journal's readership.

\section{Acknowledgments}

Funding: G Hansmann receives financial support from the German Research Foundation (DFG; HA4348/2-2 and HA4348/6-2 KFO311), the Federal Ministry of Education and Research (BMBF ViP+ program-03VP08053; BMBF $01 \mathrm{KC} 2001 \mathrm{~B})$ and the European Pediatric Pulmonary Vascular Disease Network (https://www.pvdnetwork.org/ receives financial support the Netherlands Cardiovascular Research Initiative: the Dutch Heart Foundation, Dutch Federation of University Medical Centers, the Netherlands Organization for Health Research and Development, and the Royal Netherlands Academy of Sciences (Grant 201829 awarded to the Phaedra-Impact consortium and Grant 2017-10 awarded to the DOLPHIN consortium).

\section{Footnote}

Provenance and Peer Review: This article was commissioned by the editorial office, Cardiovascular Diagnosis and Therapy for the series "Right Ventricular Dysfunction". The article did not undergo external peer review.

Conflicts of Interest: The authors have completed the ICMJE uniform disclosure form (available at http://dx.doi. org/10.21037/cdt-20-565). The series "Right Ventricular
Dysfunction" was commissioned by the editorial office without any funding or sponsorship. MK, HJB and GH served as the unpaid Guest Editors of the series. The authors have no other conflicts of interest to declare.

Ethical Statement: The authors are accountable for all aspects of the work in ensuring that questions related to the accuracy or integrity of any part of the work are appropriately investigated and resolved.

Open Access Statement: This is an Open Access article distributed in accordance with the Creative Commons Attribution-NonCommercial-NoDerivs 4.0 International License (CC BY-NC-ND 4.0), which permits the noncommercial replication and distribution of the article with the strict proviso that no changes or edits are made and the original work is properly cited (including links to both the formal publication through the relevant DOI and the license). See: https://creativecommons.org/licenses/by-nc-nd/4.0/.

\section{References}

1. Hansmann G, Koestenberger M, Alastalo TP, et al. 2019 updated consensus statement on the diagnosis and treatment of pediatric pulmonary hypertension: The European Pediatric Pulmonary Vascular Disease Network (EPPVDN), endorsed by AEPC, ESPR and ISHLT. J Heart Lung Transplant 2019;38:879-901.

2. Abman SH, Hansmann G, Archer SL, et al. Pediatric Pulmonary Hypertension: Guidelines From the American Heart Association and American Thoracic Society. Circulation 2015;132:2037-99.

3. Rosenzweig EB, Abman SH, Adatia I, et al. Paediatric pulmonary arterial hypertension: updates on definition, classification, diagnostics and management. Eur Respir J 2019;53:1801916.

4. Simonneau G, Montani D, Celermajer DS, et al. Haemodynamic definitions and updated clinical classification of pulmonary hypertension. Eur Respir J 2019;53:1801913.

5. Galiè N, McLaughlin VV, Rubin LJ, et al. An overview of the 6th World Symposium on Pulmonary Hypertension. Eur Respir J 2019;53:1802148.

6. Galiè N, Channick RN, Frantz RP, et al. Risk stratification and medical therapy of pulmonary arterial hypertension. Eur Respir J 2019;53:1801889.

7. McLaughlin VV, Hoeper MM, Channick RN, et al. Pulmonary Arterial Hypertension-Related Morbidity Is 
Prognostic for Mortality. J Am Coll Cardiol 2018;71:752-63.

8. Dandel M, Hetzer R. Echocardiographic assessment of the right ventricle: Impact of the distinctly load dependency of its size, geometry and performance. Int J Cardiol 2016;221:1132-42.

9. Vonk Noordegraaf A, Chin KM, Haddad F, et al. Pathophysiology of the right ventricle and of the pulmonary circulation in pulmonary hypertension: an update. Eur Respir J 2019;53:1801900.

10. Agrawal V, Lahm T, Hansmann G, et al. Molecular mechanisms of right ventricular dysfunction in pulmonary arterial hypertension: focus on the coronary vasculature, sex hormones, and glucose/lipid metabolis. Cardiovasc Diagn Ther 2020;10:1522-40.

11. Heinzel F, Hegemann N, Hohendanner F, et al. Left ventricular dysfunction in heart failure with preserved ejection fraction-molecular mechanisms and impact on right ventricular function. Cardiovasc Diagn Ther 2020;10:1541-60.

12. Andersen, A, van der Feen, DE, Anderson S, et al. Animal models of right heart failure. Cardiovasc Diagn Ther 2020;10:1561-79.

13. Bernardo RJ, Haddad F, Couture EJ, et al. Mechanics of right ventricular dysfunction in pulmonary arterial hypertension and heart failure with preserved ejection fraction. Cardiovasc Diagn Ther 2020;10:1580-1603.

14. Truong U, Meinel K, Haddad F, et al. Update on noninvasive imaging of right ventricle dysfunction in pulmonary hypertension. Cardiovasc Diagn Ther 2020;10:1604-24.

15. Santens B, Van De Bruaene A, De Meester P, et al. Diagnosis and treatment of right ventricular dysfunction in congenital heart disease. Cardiovasc Diagn Ther 2020;10:1625-45.

16. Sanders JL, Koestenberger M, Rosenkranz S, et al. Right ventricular dysfunction and long-term risk of death. Cardiovasc Diagn Ther 2020;10:1646-58.

17. Inampudi C, Tedford RJ, Hemnes AR, et al. Treatment of Right Ventricular Dysfunction and Heart Failure in Pulmonary Arterial Hypertension. Cardiovasc Diagn Ther 2020;10:1659-74.

18. Schweintzger S, Koestenberger M, Schlagenhauf A, et al. Safety and efficacy of the endothelin receptor antagonist macitentan in pediatric pulmonary hypertension. Cardiovasc Diagn Ther 2020;10:1675-85.

19. Giagnorio R, Hansmann G. Mineralocorticoid receptor blockade improves pulmonary hypertension and right ventricular function in bronchopulmonary dysplasia: a case report. Cardiovasc Diagnosis Ther 2020;10:1686-90.

20. Bakas AM, Healy HM, Bell KA, et al. Prenatal duct closure leading to severe pulmonary hypertension in a preterm neonate-a case report. Cardiovasc Diagnosis Ther 2020;10:1691-5.

21. Sallmon H, Berger F, Weber SC, et al. Interventional creation of an endogenous reverse Potts shunt in an infant with pulmonary hypertension and genetic surfactant disorder - a case report. Cardiovasc Diagn Ther 2020;10:1696-1700.

22. Dejea H, Bonnin A, Cook AC. Cardiac multi-scale investigation of the right and left ventricle ex vivo: a review. Cardiovasc Diagn Ther 2020;10:1701-17.

23. Hansmann G, Rich S, Maron BA. Cardiac catheterization in pulmonary hypertension: Doing it right, with a catheter on the left. Cardiovasc Diagn Ther 2020;10:1718-24.

24. Navaratnam M, DiNardo JA. Peri-operative right ventricular dysfunction-the anesthesiologist's view. Cardiovasc Diagn Ther 2020;10:1725-34.

25. Klinke A, Schubert T, Müller M, et al. Emerging Therapies for RV Dysfunction and Failure. Cardiovasc Diagn Ther 2020;10:1735-67.
Cite this article as: Koestenberger M, Bogaard HJ, Hansmann

G. Getting to the bottom of right heart failure. Cardiovasc Diagn Ther 2020;10(5):1517-1521. doi: 10.21037/cdt-20-565 